\title{
In need of constraint: Understanding the role of the cingulate cortex in the impulsive mind
}

Johannes Golchert ${ }^{1}$, Jonathan Smallwood ${ }^{3,}$, Elizabeth Jefferies ${ }^{3}$, Franziskus Liem ${ }^{1}$, Julia M. Huntenburg ${ }^{1,5}$, Marcel Falkiewicz ${ }^{1}$, Mark E. Lauckner ${ }^{1}$, Sabine Oligschläger ${ }^{1}$, Arno Villringer ${ }^{2,4}$, Daniel S. Margulies ${ }^{1, *}$

${ }^{1}$ Max Planck Research Group for Neuroanatomy \& Connectivity, Max Planck Institute for Human Cognitive and Brain Sciences, Leipzig, Germany

${ }^{2}$ Department of Neurology, Max Planck Institute for Human Cognitive and Brain Sciences and Department of Cognitive Neurology, University Hospital Leipzig, Leipzig, Germany

${ }^{3}$ Department of Psychology, University of York, Hesslington, York, United Kingdom

${ }^{4}$ Center for Stroke Research Berlin, Charité-Universitätsmedizin Berlin and Berlin School of Mind and Brain, Humboldt University, Berlin, Germany

${ }^{5}$ Neurocomputation and Neuroimaging Unit, Department of Education and Psychology, Free University of Berlin, Berlin, Germany

\section{* Correspondence:}

jonny.smallwood@york.ac.uk or margulies@cbs.mpg.de 


\section{Abstract}

Impulsive behavior often occurs without forethought and can be driven by strong emotions or sudden impulses, leading to problems in cognition and behavior across a wide range of situations. Although neuroimaging studies have explored the neurocognitive indicators of impulsivity, the large-scale functional networks that contribute to different aspects of impulsive cognition remain unclear. In particular, we lack a coherent account of why impulsivity is associated with such a broad range of different psychological features. Here, we use resting state functional connectivity, acquired in two independent samples, to investigate the neural substrates underlying different aspects of self-reported impulsivity. Based on the involvement of the anterior cingulate cortex (ACC) in cognitive but also affective processes, five seed regions were placed along the caudal to rostral gradient of the ACC. We found that positive urgency was related to functional connectivity between subgenual ACC and bilateral parietal regions such as retrosplenial cortex potentially highlighting this connection as being important in the modulation of the non-prospective, hastiness - related aspects of impulsivity. Further, two impulsivity dimensions were associated with significant alterations in functional connectivity of the supragenual ACC: (i) lack of perseverance was positively correlated to connectivity with the bilateral dorsolateral prefrontal cortex and right inferior frontal gyrus and (ii) lack of premeditation was inversely associated with functional connectivity with clusters within bilateral occipital cortex. Further analysis revealed that these connectivity patterns overlapped with bilateral dorsolateral prefrontal and bilateral occipital regions of the multiple demand network, a large-scale neural system implicated in the general control of thought and action. Together these results demonstrate that different forms of impulsivity have different neural correlates, which are linked to the functional connectivity of a region of anterior cingulate cortex. This suggests that poor perseveration and premeditation might be linked to dysfunctions in how the 
rostral zone of the ACC interacts with the multiple demand network that allows cognition to proceed in a controlled way.

Keywords: impulsivity, UPPS-P, functional connectivity, anterior cingulate cortex, multiple demand network 


\section{Introduction}

Impulsive behavior occurs when actions are "poorly conceived, prematurely expressed, unduly risky or inappropriate to the situation and often result in undesirable consequences" (Daruna and Barnes, 1993, p. 23; cf. Arce and Santisteban, 2006, Dalley et al., 2011). It is a broad construct that captures the influence of motor, cognition and emotion on behavior (see Evenden, 1999) with a wide range of functional outcomes (for reviews see: Dalley et al., 2011; Moeller et al., 2001). Different aspects of impulsivity can be assessed by questionnaires such as the UPPS impulsive behavior scale (Whiteside and Lynam, 2001) that uses a factor analytic approach to identify 4 components: (i) negative urgency, which refers to acting rashly out of a negative mood; (ii) lack of perseverance that describes circumstances of having difficulties in staying on task; (iii) lack of premeditation - reflecting the hampered ability to anticipate future consequences of an action; and (iv) sensation seeking, a tendency to seek and engage in exciting, novel and dangerous situations. Cyders and Smith (2007) added a fifth component — positive urgency - to differentiate between rash actions that occur under conditions of either negative or positive mood.

Although the construct of impulsivity as a wide ranging trait with a heterogeneous mixture of affective and cognitive factors is well established, it is unclear why such a variety of different cognitive and behavioral attributes tend to co-occur in individuals. It is possible that understanding the neurocognitive architecture that supports different types of impulsive behavior could help explain what causes this clustering of diverse symptoms under the rubric of the same broad psychological construct. To date, neurocognitive evidence has implicated alterations in how the cortex interacts with limbic and striatal systems (e.g. Brewer and Potenza, 2008; Brown et al., 2006; Dalley et al., 2011; Robbins, 2007) as well as differences in the global level of brain networks organization (Davis et al., 2013). One cortical structure that may mediate 
impulsive behavior is the anterior cingulate cortex (ACC). Recently, Casetellanos-Ryan and Seguin (2015) argued that, along with other regions of the prefrontal cortex, the ACC is important in impulsivity because of its role in many cognitive functions that seem to be altered in impulsivity. The authors argue that many of the cognitive processes that contribute to impulsive decision making and behavior involve different aspects of executive functions (such as planning, response/motor inhibition, problems in perceiving/attending environmental information, selective attention and working memory). These processes are partly mediated by the interactions between the ACC and other aspects of the PFC (for a detailed description see their review).

Studies have implicated the ACC in a wide range of different cognitive processes that could have relevance to impulsivity. These include conflict monitoring (Botvinick et al., 1999, 2001, 2004), attentional (e.g. Aarts and Roelofs, 2011; Crottaz-Herbette and Menon, 2006) and motor control (e.g. Asemi et al., 2015; Chouinard and Paus, 2006), working memory (e.g. Lenartowicz and Mclntosh, 2005), task switching (e.g. Woodward et al., 2008), adaptive choices (Economides et al., 2014) and response inhibition (Braver et al., 2001). It has also been shown to be involved in more complex social processes (Lavin et al., 2013), as well as in the regulation of emotional states (for a review see: Etkin et al., 2011) and task disengagement (Bernhardt et al., 2014). Consistent with a role in many different processes, the functional architecture of the ACC suggests it is important for integrating and processing information from different sources to guide behavior (cf. Kelly et al., 2009). It is a "cortical hub" with projections to several different brain areas both in non-humans (Devinsky et al., 1995) and humans (e.g. Hagmann et al., 2008) allowing it to integrate both bottom-up and top-down signals from different regions of cortex (e.g. Comte et al., 2014). Accordingly, various lines of research have demonstrated the existence of functionally distinct subdivisions within the ACC (e.g. Bush et al., 2000). Functional connectivity studies have shown that connectivity patterns are organized along a rostral-caudal gradient with regions in the midcingulate cortex, located caudally to the ACC, involved in 
motoric processing, more anterior regions implicated in general process of control and subgenual aspects implicated in the limbic system and default mode network (Margulies et al., 2007). It is important to note that these patterns of connectivity do not mean that subregions of the ACC serve discrete functions in all circumstances. For example, there are a number of fMRI studies showing activation associated with emotion regulation in rostral and dorsal regions of the ACC, as well as in the subgenual areas (e.g., Domes et al., 2010; Goldstein et al., 2007; Gröne et al., 2015; Ochsner and Gross, 2005; see also Vogt, 2005 for a review on ACC peak activation sites during processing of simple emotions).

The current study explores whether different aspects of impulsivity can be understood in terms of functional variation in dissociable networks anchored in the ACC. There are several different ways in which the functional architecture of the anterior cingulate could account for different aspects of impulsive behavior. One possibility is that because the anterior cingulate is itself a functionally heterogeneous region of cortex (Bush et al., 2000, Margulies et al., 2007) different cognitive features of impulsivity could depend on dysregulation in different regions of the anterior cingulate. According to this modular account, population level variation in different aspects of impulsivity would be associated with variation in the functional connectivity of different subregions of the ACC. A second possibility is that a single region of the ACC could be linked to different aspects of impulsivity by exhibiting different patterns of connectivity, each associated with a different type of impulsive behavior. Consistent with this hub view, evidence has implicated the mid-anterior cingulate in the multiple demand network (MDN), a system that plays an important role in the production of controlled cognition across a wide range of different circumstances (Duncan, 2010). According to this hub account, cross-sectional variation in different elements of impulsivity could be related to unique patterns of functional connectivity from the same region within the anterior cingulate cortex, particularly regions involved in the MDN. 
To understand how different aspects of impulsivity are related to the functional connectivity of the ACC we analyzed resting state functional connectivity data from more than 200 individuals, acquired at two different sites. In both samples, impulsivity was assessed using the UPPS-P impulsive behavior scale (Cyders and Smith, 2007) that captures different dimensions of impulsivity. We selected five caudal to rostral regions of interest reflecting its spectrum of connectivity patterns across cognitive and affective domains (Margulies et al., 2007; Kelly et al., 2009). We performed a seed-based functional connectivity analysis to examine if its connectivity profiles vary with respect to differences in cognitive or affective components of impulsivity. We were interested in whether different aspects of impulsivity are associated with dissociable patterns of functional connectivity within subregions of the anterior cingulate.

\section{Methods}

\subsection{Participants}

Two independent datasets were used in this study: (i) the Max Planck Institute sample (MPI-S), Max Planck Institute of Human Cognitive and Brain Sciences in Leipzig, Germany, and (ii) the enhanced Nathan Kline Institute-Rockland Sample (NKI-RS, Nooner et al., 2012; made publicly available as a prospective dataset through the International Neuroimaging Data-sharing Initiative). Both research projects were approved by an Ethics committee (MPI-S: medical department of University of Leipzig; NKI-RS: Nathan Kline Institute and Montclair State University).

The MPI-S dataset contained brain imaging and behavioral data from a sample of 112 healthy participants (63 female subjects) with a mean age of 26.48 ( $S D=4.73$, range: $21-44$ ) years. The data from the NKI-RS set comprised a sample of 92 (53 female) healthy subjects (age: $M=$ 
$26.89(S D=8.7)$, range: $18-44)$. The groups did not differ in terms of their respective gender distributions (Chi-square: $X^{2}(1)=0.0028, p=0.96$ ) nor with respect to age (Welch Test: $t(134,07)=0.4, p=0.69)$. All participants were recruited through advertisement, gave written informed consent, have been screened about past and present neurological or psychiatric disorders, and were monetarily reimbursed at the end of the study. In both samples, all participants were part of larger research projects and other measurements have been administered during data collection. For the present study, only the UPPS-P impulsive behavior scale (Cyders and Smith, 2007) and magnetic resonance imaging (MRI) data were analyzed.

\subsection{Measurement of trait impulsivity}

In order to assess trait impulsivity, participants of both samples filled out the UPPS-P impulsive behavior scale (Cyders and Smith, 2007; for the MPI-S cohort the questionnaire was translated into German by a qualified translator). The UPPS-P is a 59 -item self report inventory in its revised version (originally UPPS; Whiteside and Lynam, 2001) that quantifies five different aspects of impulsive behavior: (i) negative urgency, which refers to the tendency to experience strong impulses under conditions of negative affect; (ii) lack of perseverance that reflects the experience of having problems with remaining focused on a task that might be boring or too difficult; (iii) lack of premeditation that describes the tendency to engage in an act without reflecting the consequences of that act beforehand; (iv) sensation seeking that further comprises two aspects: (a) the propensity to enjoy and chase exciting activities; (b) an openness to engage in new experiences that might be dangerous; and ( $v$ ) positive urgency that involves the tendency towards rash actions in response to very positive mood. Each item can be scored on a four-point Likert scale, ranging from 1 (strongly agree) to 4 (strongly disagree). Reversed items are recoded afterwards so that higher scores indicate a more pronounced level of self-reported trait impulsivity. In both samples, reliability analyses revealed an adequate level of internal consistency (Cronbach's alpha) for each of the five subscales (see Table 1). 


\subsection{MRI scan acquisition}

MPI-S data were acquired on a 3T Siemens Magnetom Verio Scanner and included four resting state functional magnetic resonance imaging ( $\mathrm{rs}-\mathrm{fMRI}$ ) sequences as well as an anatomical T1weighted scan. The rs-fMRI data were recorded using a BOLD-weighted multiband EPI sequence (Feinberg et al., 2010; Moeller et al., 2010; TR = $1400 \mathrm{~ms}$, TE = $39.4 \mathrm{ms,} \mathrm{flip} \mathrm{angle} \mathrm{=}$ $69^{\circ}$, multiband acceleration factor $=4$, voxel size $=2.3 \mathrm{~mm}$ isotropic, 64 slices, 657 volumes, duration $=15.30 \mathrm{~min})$. A high-resolution anatomical image was additionally acquired using a MP2RAGE sequence (Marques et al. 2010; TR = $5000 \mathrm{~ms}, \mathrm{TE}=2.92 \mathrm{~ms}, \mathrm{Tl} 1=700 \mathrm{~ms}$, $\mathrm{Tl} 2=$ $2500 \mathrm{~ms}$, flip angle $1=4^{\circ}$, flip angle $2=5^{\circ}$, voxel size $=1.0 \mathrm{~mm}$ isotropic, duration $=8.22 \mathrm{~min}$ ).

NKI-RS data were acquired on a 3T Siemens Trio Scanner, and comprised a rs-fMRI scan acquired with a BOLD-weighted multiband EPI sequence (TR $=645 \mathrm{~ms}$, TE $=30 \mathrm{~ms}$, flip angle $=60^{\circ}$, multiband acceleration factor $=4$, voxel size $=3 \mathrm{~mm}$ isotropic, 40 slices, 900 volumes, duration $=10 \mathrm{~min}$.$) and \mathrm{a}$ high resolution anatomical T1-weighted image acquired with a MPRAGE sequence $\left(T R=1900 \mathrm{~ms}, \mathrm{TE}=2.52 \mathrm{~ms}\right.$, flip angle $=9^{\circ}$, voxel size $=1.0 \mathrm{~mm}$ isotropic). In both rs-fMRI assessments subjects were instructed to keep their eyes open and to focus on a fixation cross (Nooner et al., 2012)

\subsection{Preprocessing}

All preprocessing steps were streamlined using Nipype (Gorgolewski et al., 2011), incorporating tools from FreeSurfer (Dale et al., 1999; Fischl et al., 1999), FSL (Jenkinson et al., 2012), AFNI (Cox, 1996), ANTs (Avants et al., 2011), CBS Tools (Bazin et al., 2014) and Nitime (Rokem et al., 2009). Reproducible workflows containing all implementation details for both the MPI-S ${ }^{1}$ and

\footnotetext{
${ }^{1}$ https://github.com/NeuroanatomyAndConnectivity/pipelines/blob/master/src/lsd_lemon/lsd_resting.py
} 
$\mathrm{NKI}-\mathrm{RS}^{2}$ data are available online. In brief, structural preprocessing was implemented by masking the background of the T1-weighted images using CBS Tools before volumetric segmentation and surface reconstruction with FreeSurfer's recon-all pipeline. A spatial transformation between the individual structural space and MNI152 standard space was calculated using diffeomorphic nonlinear registration implemented in ANTs SyN.

Resting state preprocessing of the MPI-S data included the following steps: (i) removing the first five EPI volumes from each resting-state scan to allow for signal equilibration; (ii) motion correction; (iii) distortion correction; (iv) coregistration to the individual T1-weighted image; (v) denoising via two steps: a) the 6 motion regressors and first derivatives, motion and signalintensity outliers (based on Nipype rapidart interface) as well as linear and quadratic trends were removed in a general linear model (GLM); b) the residual time series was used to derive 6 principal components most representative of physiological noise using the aCompCor method within white matter and CSF (Behzadi et al., 2007). These are removed along with the initial regressors in a second GLM; (vi) band pass temporal filtering $(0.01-0.1 \mathrm{~Hz})$ and mean centering as well as variance normalization of the denoised timeseries using Nitime; and (vii) spatial normalization to MNI152 standard space (2 mm isotropic) using the transformation derived during structural preprocessing. The NKI-RS data were preprocessed in a similar way with the following exceptions: (1) distortion correction was not performed as no field maps were acquired; (2) denoising was carried out in one step using the Friston 24-parameter-model (Friston et al., 1996) as implemented in C-PAC: 5 principal components (aCompCor), motion and signal-intensity outliers as well as linear and quadratic trends were regressed out; and (3) spatial normalization towards MNI152 standard space was performed with a resulting resolution of $3 \mathrm{~mm}$ isotropic. The lack of distortion correction within the NKI-RS data may impact on results within regions sensitive to susceptibility artifacts, such as orbital and ventral anterior temporal

\footnotetext{
${ }^{2}$ https://github.com/NeuroanatomyAndConnectivity/nki_nilearn/blob/master/preprocessing_pipeline.py
} 
regions. However, the location of the significant results reported below are far from these regions, indicating this preprocessing difference is not a confound for the current results. The difference in the denoising steps between the two site constitutes minor deviations in preprocessing, and the robustness of the significant results to differences in preprocessing provides further support for our cross-site validation. Finally, the differences in sampling the data to standard space ( 2 and $3 \mathrm{~mm}$ ) was established with respect to the acquisition voxel size, and should not impact on the significance thresholds used below.

\subsection{First level analysis}

Five seed regions were selected along the dorsal to caudal gradient of the ACC (Margulies et al., 2007; Kelly et al., 2009). Spherical regions of interest (ROIs) with a radius of $4 \mathrm{~mm}$ were bilaterally placed at the following coordinates (MNI): seed $s 1( \pm 5,-10,47)$, seed $s 3( \pm 5,14,42)$, seed $s 5( \pm 5,34,28)$, seed $s 7( \pm 5,47,11)$, seed i9 $( \pm 5,25,-10)$ (see Figure 1 ; coordinates and nomenclature taken from Margulies et al., 2007). The time series of all voxels within these regions were then extracted and averaged. For each dataset, the subject specific correlation between the averaged seed time series and every other voxel in the brain was calculated. The correlation maps were calculated using AFNI's 3dfim+ command. The individual correlation maps were then Fisher r-to-z transformed and smoothed with a Gaussian kernel of $6 \mathrm{~mm}$. Because the MPI-S dataset comprises four resting state scans, we ran this individual seedbased functional connectivity analysis for each scan separately and then averaged the four smoothed correlation maps. Correlation maps of the NKI-RS data were upsampled into $2 \mathrm{~mm}$ voxel space to allow for comparison between both samples. As before, all steps were implemented using Nipype (Gorgolewski et al., 2011). In order to allow for comparison between datasets, correlation maps were normalized (z-scored) separately for each individual prior to the 
group-level analysis. That is, the correlation maps were standardized voxelwise across participants, a step that allows the connectivity from different datasets to be compared.

\subsection{Group level analysis}

Higher-level ordinary least squares multiple regression analysis was performed using FMRIB's Local Analysis of Mixed Effects (FLAME OLS, as implemented in FSL FEAT). In order to increase the statistical power of our model, both datasets were simultaneously entered into the analyses. The five impulsivity subscales were used as covariates of interest, while controlling for age, sex, in-scanner motion (mean framewise displacement (Power et al., 2012)) and dataset membership. All covariates were z-scored (except for sex, which was demeaned) across the two datasets. The concatenated connectivity maps for each region of the ACC were the dependent variables. One model was run per seed region. For each impulsivity subscale, a positive and a negative contrast has been computed, were the positive contrast highlights regions whose connectivity with the seed region are positively associated with the measure of impulsivity, and the negative contrasts highlight regions showing lower connectivity with the seed region with increasing impulsivity. In addition, we also tested for interactions of the slopes between groups (F-test). Following Eklund et al. (2016), spatial maps were thresholded at $z=$ 3.1 and we selected an alpha value that corrected for the number of voxels in the brain, the number of the seed regions and the two tailed nature of the comparisons $(p<0.05$ FWE $/ 10=p$ $<0.005)$. Thresholded z-stat maps of each seed's functional connectivity pattern as modulated by each respective impulsivity dimension were derived by this analysis. The unthresholded maps for all the analyses reported in this paper are available at Neurovault at the following URL: neurovault.org/collections/MXBTLTPY/

\section{Results}

\subsection{Self-reported impulsivity}


For both datasets internal consistency for the five impulsivity subscales are presented in Table

1. The subscales had acceptable psychometric properties across both samples (all alphas > 0.74). The correlations between each of the components of impulsivity are presented in Table 2. Consistent with previous research, especially positive and negative urgency were highly correlated, whereas sensation seeking was not correlated with a lack of perseverance in both samples (e.g. Cyders and Smith, 2007). In addition, a lack of premeditation was correlated with both sensation seeking and positive urgency in the MPI-S dataset and sensation seeking and negative urgency were correlated in the NKI-RS data.

Table1.

Psychometric properties of the five impulsivity subscales

\begin{tabular}{|c|c|c|c|c|c|c|c|c|c|c|c|}
\hline & \multicolumn{6}{|c|}{ MPI-S } & \multicolumn{5}{|c|}{ NKI-RS } \\
\hline & $\mathrm{N}$ & $M$ & SD & SK & KT & $\alpha$ & $M$ & SD & SK & $\mathrm{KT}$ & $\alpha$ \\
\hline NU & 12 & 2.23 & 0.49 & -0.05 & -0.44 & 0.85 & 1.98 & 0.53 & 0.65 & 0.41 & 0.85 \\
\hline PSV & 10 & 1.95 & 0.47 & 0.38 & -0.16 & 0.85 & 1.74 & 0.41 & 0.21 & -0.47 & 0.77 \\
\hline PMD & 11 & 2.07 & 0.37 & -0.06 & -0.45 & 0.74 & 1.74 & 0.37 & 0.20 & 0.31 & 0.78 \\
\hline SS & 12 & 2.73 & 0.59 & -0.14 & -0.56 & 0.85 & 2.75 & 0.65 & -0.40 & -0.45 & 0.86 \\
\hline PU & 14 & 1.88 & 0.50 & 0.18 & -0.84 & 0.92 & 1.59 & 0.52 & 0.85 & -0.07 & 0.91 \\
\hline
\end{tabular}

Table 2.

Pearson product-moment correlations of the five impulsivity subscales 


\begin{tabular}{|c|c|c|c|c|c|c|c|c|c|c|}
\hline & NU & PSV & PMD & SS & PU & $\mathrm{NU}$ & PSV & PMD & SS & PU \\
\hline NU & - & $0.45^{\star *}$ & $0.20^{*}$ & 0.12 & $0.70^{* *}$ & - & $0.32^{* *}$ & $0.29^{* *}$ & $0.30^{* *}$ & $0.69^{* *}$ \\
\hline PSV & & - & $0.33^{* *}$ & -0.05 & $0.46^{* *}$ & & - & $0.35^{\star *}$ & -0.04 & $0.32^{* *}$ \\
\hline PMD & & & - & $0.37^{* *}$ & $0.34^{* *}$ & & & - & 0.10 & 0.19 \\
\hline SS & & & & - & 0.13 & & & & - & $0.34^{* *}$ \\
\hline PU & & & & & - & & & & & - \\
\hline
\end{tabular}

Note: ${ }^{*} p<0.05,{ }^{* *} p<0.01,2$-tailed

$\mathrm{NU}=$ negative urgency, $\mathrm{PSV}=$ lack of perseverance, $\mathrm{PMD}=$ lack of premeditation, $\mathrm{SS}=$ sensation seeking, $\mathrm{PU}=$ positive urgency

\subsection{Functional connectivity}

We calculated the group-level connectivity maps for each seed and cohort (Figure 1). Consistent with prior work we found a shift in connectivity along the rostral-caudal gradient of the ACC (Margulies et al., 2007). The posterior seed regions showed strong connectivity with unimodal cortex in primary motor and occipital cortex, and reduced connectivity with the medial prefrontal cortex. The most anterior seeds showed the reverse pattern since it was positively coupled to the default mode network. This pattern is consistent with prior studies and is present in both samples.

\subsection{Relation to impulsivity}

We explored the relationship between the connectivity of seed regions and levels of impulsivity using a series of multiple regressions. The dependent measure was the connectivity of the seed region and the independent variable was the participants' score on different aspects of impulsivity. Separate models were calculated for each seed region. 
For the impulsivity subscale positive urgency, subgenual ACC (seed i9) negatively related to bilateral parietal regions encompassing inferior parts of the precuneus, retrosplenial cortex and intracalcarine sulcus $(r=-0.2 ; p<0.01)$. Specifically, we found that weaker coupling between the parietal regions and the subgenual ACC was related to an increased tendency to engage in impulsive actions when feeling especially positive (Figure 2).

Importantly, the most centrally located seed s5 yielded two significant associations with impulsivity: we observed a positive association between connectivity in this region and clusters in left and right middle frontal gyrus/right inferior frontal gyrus with increasing reports of difficulties in perseverance $(r=0.31, p<0.01$; Figure 3$)$. We plotted the data for each participant at each site and it can be seen that this relationship is consistent across datasets. Individuals with difficulties in maintaining focus show stronger coupling between supragenual cingulate with the prefrontal cortex (Figure 4). We also found a negative association between reports of problems in premeditation and connectivity between the same s5 seed region and clusters in bilateral medial occipital cortex, extending into regions of lateral occipital, occipitotemporal-fusiform cortex $(r=-0.23, p<0.01)$. This association was comparable in the two samples (Figure 3 and 4). Individuals who engaged in actions without sufficient forethought have lower coupling between supragenual cingulate cortex and medial as well as lateral parts of occipital cortex. Notably, despite both deficits in premeditation and perseveration showing a relationship to the functional connectivity of the same region of $A C C$, we found no evidence of a reciprocal relationship (i.e. perseveration was not related to the connectivity in the cluster associated with premeditation and vice versa; see Figure 4). We did not detect any significant interactions in the areas reported. We observed no significant association between the impulsivity dimensions and the other seeds.

\subsection{Similarities to the Multiple Demand Network}


The s5 seed revealed a pattern of connectivity that linked impulsivity to a network that encompassed lateral frontal, occipital and parietal regions. These regions are members of the multiple demand network (MDN). Therefore, we explored whether the observed patterns of functional connectivity associated with perseveration and premeditation captured regions of this network. We compared the spatial distribution of seed s5 connectivity associated with poor premeditation and perseverance with a spatial map of the MDN (from Fedorenko et al., 2013; see Figure 5) and found evidence of substantial overlap. The connectivity patterns from seed s5 associated with poor perseverance show overlap in the inferior frontal sulcus in the left hemisphere and in the inferior frontal junction in both hemispheres. The weaker connections to occipital and parietal regions related to difficulties in premeditation overlapped with the MDN bilaterally in regions of lateral occipital cortex as well as in dorsal regions of the parietal cortex. Also, we observed that the seed region fell at the ventral anterior boundary of this network (see grey panel). Thus, different patterns of connectivity associated with problems in task focus and planning were related to dissociable patterns of connectivity within a large-scale network associated with careful cognition and behavior (Duncan, 2010).

\section{Discussion}

Our study set out to understand whether functional connectivity patterns of the ACC could explain why heterogeneous cognitive and behavioral problems tend to cluster together in individuals with impulsivity. Across two independent samples, we found evidence that problems with perseverance and premeditation are associated with independent variations in the functional connectivity of the supragenual cingulate. Reports of increased difficulties in sustaining focus were related to increased functional coupling between the supragenual cingulate (seed s5) and the bilateral regions of lateral prefrontal cortex. In contrast, neglecting the future consequences of an action was associated with decreased functional connectivity 
between the same seed and bilateral regions of the occipital and parietal cortex. Finally, positive urgency was associated with weaker connectivity between the subgenual cingulate (seed i9) and bilateral regions of medial parietal cortex. Together, these results indicate that the connectivity of the ACC at rest varies with respect to different aspects of impulsivity, a pattern that suggests that this region may be important in explaining why different constructs often cooccur together in impulsive individuals.

Our data suggest that dysfunctions in a central region of the cingulate cortex are associated with a variety of different aspects of the psychological features that are associated with impulsivity. We found that a lack of perseverance relates to increased functional communication between the rostral supragenual ACC and regions of the dorsolateral prefrontal cortex. Lateral prefrontal regions have been implicated in impulsive behavior previously (Aron et al., 2003; Ding et al., 2014; Weafer et al., 2015; Winstanley et al., 2006) and more generally in goal maintenance and pursuit (Berkman et al., 2012). Since the supragenual ACC is implicated in evaluative processes such as conflict monitoring (e.g. Botvinick et al., 2004), the observed elevated connectivity between that region and dorsolateral prefrontal cortex might reflect an overly excessive coupling between conflict monitoring and goal maintenance systems. This in turn could result in a failure to sustain attention as individuals may switch between different goal states. Problems in premeditation were associated with reduced connectivity between the same rostral supragenual region of ACC and medial/lateral regions of the visual cortex, including fusiform, medial and lateral occipito-temporal cortex. These regions not only play important roles in extracting higher order information from sensory input (for a review see e.g. GrillSpector and Malach, 2004), but are also modulated by attention (e.g. Woldorff et al., 2004). For instance, calculating effective connectivity, Crottaz-Herbette \& Menon (2006) demonstrated increased effects of the ACC on striate regions during a visual oddball task supporting the ACCs role in attentional modulation during sensory processing. Since attentional control underlies the 
capacity to follow up on goals despite distractions (cf. Aarts \& Roelofs, 2011), it is possible that the observed reductions in connectivity reflects a situation in which distracting environmental information are insufficiently suppressed at the moment when an action is initiated. This would support claims that impulsive behavior is initiated under conditions when sensory evidence has been inadequately sampled ("reflection impulsivity", Kagan, 1966; cf. Dalley et al., 2011; Evenden, 1999), and could explain why impulsive individuals are often unduly influenced by distractor processes (Yantis et al., 2012) making it difficult to keep track of specific goals. Finally, we also found that positive urgency related to decreased functional connectivity between the subgenual ACC and bilateral parietal regions such as retrosplenial cortex. These regions fall within the so-called default mode network (DMN), a system that is known to be important in envisioning the future (Schacter and Addis, 2007). Studies have suggested that imagining future outcomes helps participants to make more effective long term decisions (Peters and Büchel, 2010). One possible interpretation of the data associating the connectivity of the subgenual ACC with retrosplenial cortex is that it describes an absence of effective prospection that leads to the rash behavior that can characterize the trait of positive urgency. Regardless of the precise psychological mechanisms through which the observed patterns of connectivity contribute to different types of impulsivity, the fact that dissociable patterns of functional connectivity associated with distinct aspects of impulsivity emerge from the same region of cingulate cortex indicates that the rostral supragenual ACC is an important hub regions whose functional connectivity provides important insights into the cognitive processes that underpin impulsivity.

The observed patterns of connectivity also provide a possible explanation why impulsivity is associated with problems in cognition and behavior across a wide range of situations. We found that two types of impulsivity emerged from differences in cingulate connectivity with a distributed set of regions across the cortex that are known to be important across a range of different task 
conditions - the multiple demand network (MDN; Duncan, 2010). Since the MDN network is defined through its importance in a wide range of different situations (Fedorenko et al., 2013), our demonstration that its communication with a core hub in the cingulate cortex becomes deregulated in impulsivity might explain why problems in cognition and behavior emerge across a range of situations. Problems linked to impulsivity, such as the lacking ability to think ahead, or to focus on a task, have a pervasive influence on behavior since they reflect dysfunctions in communication between an important cortical hub and a neurocognitive system that support thought, cognition and action in a domain general manner. Although our study highlights the visual similarity between the MDN and the functional patterns associated with impulsivity, this does not constitute a formal statistical link. Building on our observation, future work could profitably explore the resting state functional connectivity patterns associated with impulsivity and the neural activation that is common to many different difficult tasks, either in a large scale study or through a formal meta-analysis using tools such as Neurosynth (Yarkoni et al., 2011). Indeed, a recent meta-analysis has highlighted the viability of understanding complex traits like impulsivity through their shared links to self-report and tasks (Sharma et al., 2014).

\section{Limitations}

The main focus of our study was to explore whether several personality facets related to impulsive behavior can be explained by dissociable functional networks anchored along the caudal-rostral gradient of the ACC. Although our results support this hypothesis the correlational nature of the analyses performed limits the interpretational framework by making it impossible to infer any causal or hierarchical relationship. From a mechanistic point of view, this leaves open questions about the origin of inter-individual differences in those traits with respect to functional connectivity. Along similar lines, we restricted our analyses to connectivity profiles of the anterior cingulate cortex by using an a priori analytical approach, which, although appropriate with respect to our circumscribed hypotheses, prevents the identification of any association 
"outside" the ACC. It is important to note that while the ACC is important for impulsivity (Castellanos-Ryan \& Seguin, 2015) there is evidence that other cortical regions such as ventromedial prefrontal cortex, orbitofrontal cortex, presupplementary motor area or dorsolateral prefrontal cortex, but also midbrain (limbic, striatal) regions are similarly important for understanding the various aspects of impulse control (see e.g., Li et al., 2013; Robbins et al., 2012; Shannon et al., 2011). Indeed, studies suggest that the interaction between these two systems is key to understanding the manifoldness of the construct (e.g. Dalley et al., 2011, cf. Castellanos-Ryan \& Seguin, 2015). Although this study might be the first examining different impulsiveness-related personality traits in terms of their underlying intrinsic functional brain organization using rs-fMRI in healthy adults, we encourage future studies to investigate those traits by using more fine grained and data-driven methods that target the whole brain instead of specific regions of interest. Lastly, although our analysis highlights the ACC as important in the genesis of impulsive behavior, our analysis does not support a detailed mapping between the cognitive processes that are characterized by our functional connectivity evidence. For example, it is possible that a single cognitive process is represented in the s5 region of the ACC and that the manner that this function expresses itself in its interactions with other regions of cortex determines whether behavior is characterized as lacking perseverance or premeditation. Alternatively, s5 may reflect the convergence of different types of cognitive and emotional processing that are themselves represented in other cortical regions. In contrast to our lack of detail at the psychological level, our data does allow us to make precise statements at the level of functional connectivity because the s5 seed of the anterior cingulate cortex is able to discriminate between different facets of impulsive behavior. Building on our observations that the anterior cingulate, and in particular the s5 region, are important in different aspects of impulsivity, we hope that future studies will be able to specify the psychological functions that this region supports, and its role in impulsivity, with greater detail. 


\section{Conclusions and future directions}

Using a large sample of participants recruited from two different countries, we found consistent evidence that different types of impulsivity are reflected by different patterns of functional coupling between regions in ACC with lateral and medial parietal cortex, as well as lateral frontal cortex. The observed connectivity was topographically consistent with the distribution of the MDN (Duncan, 2010) - a system important for the general control of thought and action. The observed association with the MDN helps to explain why impulsive behavior tends to occur in many different situations. Interestingly, we could differentiate forms of impulsivity based on the functional coupling of the supragenual seed $s 5$, a region on the boundary of the MDN, rather than seed s3, a region within the network itself. This suggests that problems may be in the integration between the MDN and other large scale systems. Both task disengagement (Christoff et al., 2009; Mason et al., 2007) and future planning (Schacter and Addis, 2007) have been linked to the $\mathrm{DMN}$, and so it is possible that our findings reveal that the cognitive problems associated with impulsivity emerge from dysfunctions in interactions between the MDN and DMN. As seed s5 marks the transition between regions in the cingulate cortex coupled to the DMN and those that are not, this could explain why this region of cortex seems especially important in impulsivity. Based on these findings, we hypothesize that certain aspects of impulsivity emerge from dysfunctions in the way the cingulate cortex controls interactions between the DMN and MDN. This hypothesis could further be explored by assessing how the integrity of the white and grey matter of these regions is linked both to traits of impulsivity and their cognitive manifestations (poor planning and task disengagement). More generally, our study provides evidence that several important aspects of impulsivity can be related to the behavior of the anterior cingulate cortex at rest, an observation that means in the future researchers may be able to use measures of functional connectivity from the anterior cingulate as an additional outcome measure for evaluating drug and psychological interventions for individuals with problems in impulsivity. 
Acknowlegements. EJ was supported by grants from BBSRC (BB/J006963/1) and the European Research Council (SEMBIND - 283530). FL was supported by the Swiss National Science Foundation (P2ZHP1_155200). JS was supported by the European Research Council (WANDERINGMINDS - 646927). This publication was also made possible through the support of a grant from the John Templeton Foundation, "Prospective Psychology Stage 2: A Research Competition" to Martin Seligman. The opinions expressed in this publication are those of the author(s) and do not necessarily reflect the views of the John Templeton Foundation. 


\section{Figure Captions}

Figure 1 Group functional connectivity maps for each of the five regions of the anterior cingulate cortex $(A C C)$

The right panel illustrates the seed locations within the ACC as well as the exact coordinates (below; Figure adapted from Margulies, 2007). The left panel presents a tabular overview of the group functional connectivity maps for each seed separately for the two samples (MPI-S dataset, NKI-RS dataset) used in this study.

Acronyms: MPI-S - Max Planck Institute Sample, NKI-RS - Nathan Kline Institute Rockland Sample

Figure 2 Positive Urgency varies with functional connectivity of the subgenual region of the anterior cingulate cortex (ACC)

The figure represents the cluster corrected maps visualizing the characteristics of the functional connectivity profile of the seed region i9 as altered by positive urgency. Across two independent datasets, functional connectivity between the ACC seed and regions within the bilateral parietal cortex were negatively predicted by positive urgency. The scatterplot displays the averaged functional connectivity values extracted from that cluster and plotted against the individual selfreport scores separately for each dataset. Spatial maps were thresholded using FSL's easythresh: $Z=3.1$, FWE $p<0.005)$.

Figure 3 Lack of perseverance and lack of premeditation differently modulate functional connectivity patterns of the most rostral supragenual region of the anterior cingulate cortex The upper panel of the figure depicts the cluster corrected maps of the functional connectivity profile of the supragenual seed region s5 that covaries with lack of perseverance: two significant clusters within the bilateral dorsolateral prefrontal cortex (red) were derived by the analysis 
across two independent datasets. The lower panel shows the corresponding results for lack of premeditation: Two significant clusters within the bilateral occipital cortex (blue) were obtained by this analysis. Spatial maps were thresholded using FSL's easythresh: $Z=3.1$, FWE $p<$ 0.005).

Figure 4 Scatterplots demonstrating the linear relation between extracted functional connectivity values and behavioral self-report scores.

The left panel depicts the two different connectivity profiles of supragenual seed region s5 as modulated by lack of perseverance and lack of premeditation (cf. Figure 2). The scatterplots show the averaged functional connectivity values extracted from these clusters plotted against the self-report scores for each participant, separately for each dataset. In order to demonstrate the independence of the results, we also plotted the opposite behavioral scores against the cluster values (e.g. lack of premeditation scores against clusters derived by lack of perseverance and vice versa).

Figure 5 Clusters associated with a lack of perseverance and premeditation overlap with the multiple demand network

This figure illustrates the overlap of the the seed-based functional connectivity results for lack of perseverance (red) and lack of premeditation (blue) and the pattern of the multiple demand network (green; from Fedorenko et al., 2013) proposed by Duncan (2010). Overlaps in bilateral dorsolateral prefrontal cortex as well as in bilateral occipital cortex are indicated in yellow and light blue, respectively. These maps are fully saturated to maximize the visibility of spatial overlap. 


\section{References}

Aarts, E., Roelofs, A., 2011. Attentional control in anterior cingulate cortex based on probabilistic cueing. J. Cogn. Neurosci. 23, 716-727. doi:10.1162/jocn.2010.21435

Arce, E., Santisteban, C., 2006. Impulsivity: A review. Psicothema 18, 213-220.

Aron, A.R., Fletcher, P.C., Bullmore, E.T., Sahakian, B.J., Robbins, T.W., 2003. Stop-signal inhibition disrupted by damage to right inferior frontal gyrus in humans. Nat. Neurosci. 6, 115-116. doi:10.1038/nn1003

Asemi, A., Ramaseshan, K., Burgess, A., Diwadkar, V. a., Bressler, S.L., 2015. Dorsal anterior cingulate cortex modulates supplementary motor area in coordinated unimanual motor behavior. Front. Hum. Neurosci. 9, 1-10. doi:10.3389/fnhum.2015.00309

Avants, B.B., Tustison, N.J., Song, G., Cook, P.A., Klein, A., Gee, J.C., 2011. A reproducible evaluation of ANTs similiarity metric performance in brain image registration. Neuroimage 54, 2033-2044. doi:10.1016/j.neuroimage.2010.09.025

Bazin, P.L., Weiss, M., Dinse, J., Schäfer, A., Trampel, R., Turner, R., 2014. A computational framework for ultra-high resolution cortical segmentation at 7Tesla. Neuroimage 93, 201209. doi:10.1016/j.neuroimage.2013.03.077

Behzadi, Y., Restom, K., Liau, J., Liu, T.T., 2007. A component based noise correction method (CompCor) for BOLD and perfusion based fMRI. Neuroimage 37, 90-101. doi:10.1016/j.neuroimage.2007.04.042

Berkman, E.T., Falk, E.B., Lieberman, M.D., 2012. Interactive effects of three core goal pursuit processes on brain control systems: Goal maintenance, performance monitoring, and response inhibition. PLoS One 7, e40334. doi:10.1371/journal.pone.0040334 
Bernhardt, B.C., Smallwood, J., Tusche,. A., Ruby, F.J., Engen, H.G., Steinbeis, N., Singer, T., 2014. Medial prefrontal and anterior cingulate cortical thickness predicts shared individual differences in self-generated thought and temporal discounting. Neuroimage 90, 290-297. doi:10.1016/j.neuroimage.2013.12.040

Botvinick, M.M., Braver, T.S., Barch, D.M., Carter, C.S., Cohen, J.D., 2001. Conflict monitoring and cognitive control. Psychol. Rev. 108, 624-652. doi:10.1037//0033-295X.I08.3.624

Botvinick, M.M., Cohen, J.D., Carter, C.S., 2004. Conflict monitoring and anterior cingulate cortex: An update. Trends Cogn. Sci. 8, 539-546. doi:10.1016/j.tics.2004.10.003

Botvinick, M.M., Nystrom, L.E., Fissell, K., Carter, C.S., Cohen, J.D., 1999. Conflict monitoring versus selection-for-action in anterior cingulate cortex. Nature 402, 179-181. doi:10.1038/46035

Braver, T.S., Barch, D.M., Gray, J.R., Molfese, D.L., Snyder, A., 2001. Anterior cingulate cortex and response conflict: effects of frequency, inhibition and errors. Cereb. Cortex 11, 825836. doi:10.1093/cercor/11.9.825

Brewer, J.A., Potenza, M.N., 2008. The neurobiology and genetics of impulse control disorders: Relationships to drug addictions. Biochem. Pharmacol. 75, 63-75. doi:10.1016/j.bcp.2007.06.043

Brown, S.M., Manuck, S.B., Flory, J.D., Hariri, A.R., 2006. Neural basis of individual differences in impulsivity: contributions of corticolimbic circuits for behavioral arousal and control. Emotion 6, 239-245. doi:10.1037/1528-3542.6.2.239

Bush, G., Luu, P., Posner, M., 2000. Cognitive and emotional influences in anterior cingulate cortex. Trends Cogn. Sci. 4, 215-222. doi:10.1016/S1364-6613(00)01483-2 
Casetellanos-Ryan, N., Seguin, J.R., 2015. Prefrontal and Anterior Cingulate Cortex Mechanisms of Impulsivity, in: Beauchaine, T.P., Hinshaw, S.P. (Eds.), The Oxford Handbook of Externalizing Spectrum Disorders. Oxford University Press, pp. 201-219. doi:10.1093/oxfordhb/9780199324675.013.13

Chouinard, P.A., Paus, T., 2006. The primary motor and premotor areas of the human cerebral cortex. Neurosci. 12, 143-152. doi:10.1177/1073858405284255

Christoff, K., Gordon, A.M., Smallwood, J., Smith, R., Schooler, J.W., 2009. Experience sampling during $\mathrm{fMRI}$ reveals default network and executive system contributions to mind wandering. Proc. Natl. Acad. Sci. U. S. A. 106, 8719-8724. doi:10.1073/pnas.0900234106

Comte, M., Schön, D., Coull, J.T., Reynaud, E., Khalfa, S., Belzeaux, R., Ibrahim, el C., Guedj, E., Blin, O., Weinberger, D.R., Fakra, E., 2014. Dissociating Bottom-Up and Top-Down Mechanisms in the Cortico-Limbic System during Emotion Processing. Cereb. Cortex 26, 144-155. doi:10.1093/cercor/bhu185

Cox, R.W., 1996. AFNI: software for analysis and visualization of functional magnetic resonance neuroimages. Comput. Biomed. Res. 29, 162-173. doi:10.1006/cbmr.1996.0014

Crottaz-Herbette, S., Menon, V., 2006. Where and when the anterior cingulate cortex modulates attentional response: combined fMRI and ERP evidence. J. Cogn. Neurosci. 18, 766-80. doi:10.1162/jocn.2006.18.5.766

Cyders, M. A., Smith, G.T., 2007. Mood-based rash action and its components: Positive and negative urgency. Pers. Individ. Dif. 43, 839-850. doi:10.1016/j.paid.2007.02.008

Dale, A.M., Fischl, B., Sereno, M.I., 1999. Cortical surface-based analysis. I. Segmentation and surface reconstruction. Neuroimage 9, 179-194. doi:10.1006/nimg.1998.0395 
Dalley, J.W., Everitt, B.J., Robbins, T.W., 2011. Impulsivity, Compulsivity, and Top-Down Cognitive Control. Neuron 69, 680-694. doi:10.1016/j.neuron.2011.01.020

Daruna, J.H., Barnes, P.A., 1993. A neurodevelopmental view of impulsivity, in: McCown, W.G., Johnson, J.L., Shure, M.B. (Eds.), The impulsive Client: Theory, Research and Treatment. American Psychological Association, Washington, D.C., pp. 23-27. doi:http://dx.doi.org/10.1037/10500-000

Davis, F.C., Knodt, A.R., Sporns, O., Lahey, B.B., Zald, D.H., Brigidi, B.D., Hariri, A.R., 2013. Impulsivity and the modular organization of resting-state neural networks. Cereb. Cortex 23, 1444-1452. doi:10.1093/cercor/bhs126

Devinski, O., Morrell, M.J., Vogt, B.A., 1995. Contributions of the anterior cingulate cortex to behaviour. Brain 118, 279-306. doi:10.1093/brain/118.1.279

Ding, W-N., Sun, J-H., Sun, Y-W., Chen, X., Zhou, Y., Zhuang, Z-G., Li, L., Zhang, Y., Xu, J-R., Du, Y-S., 2014. Trait impulsivity and impaired prefrontal impulse inhibition function in adolescents with internet gaming addiction revealed by a Go/No-Go fMRI study. Behav. Brain Funct. 10, 20. doi:10.1186/1744-9081-10-20

Domes, G., Schulze, L., Böttger, M., Grossmann, A., Hauenstein, K., Wirtz, P.H., Heinrichs, M., Herpertz, S.C., 2010. The neural correlates of sex differences in the emotional reactivity and emotion regulation. Hum. Brain Mapp. 31, 758-769. doi:10.1002/hbm.20903

Duncan, J., 2010. The multiple-demand (MD) system of the primate brain: mental programs for intelligent behaviour. Trends Cogn. Sci. 14, 172-179. doi:10.1016/j.tics.2010.01.004

Economides, M., Guitart-Masip, M., Kurth-Nelson, Z., Dolan, R.J., 2014. Anterior Cingulate Cortex Instigates Adaptive Switches in Choice by Integrating Immediate and Delayed Components of Value in Ventromedial Prefrontal Cortex. J. Neurosci. 34, 3340-3349. doi:10.1523/JNEUROSCI.4313-13.2014 
Eklund, A., Nichols, T.E., Knutsson, H., 2016. Cluster failure: Why fMRI inferences for spatial extent have inflated false-positive rates. Proc. Natl. Acad. Sci. U. S. A 113, 7900-7905. doi:10.1073/pnas.1602413113

Etkin, A., Egner, T., Kalisch, R., 2011. Emotional processing in anterior cingulate and medial prefrontal. Trends Cogn. Sci. 15, 85-93. doi:10.1016/j.tics.2010.11.004

Evenden, J.L., 1999. Varieties of impulsivity. Psychopharmacology (Berl). 146, 348-361. doi:10.1007/PL00005481

Fedorenko, E., Duncan, J., Kanwisher, N., 2013. Broad domain generality in focal regions of frontal and parietal cortex. Proc. Natl. Acad. Sci. U. S. A. 110, 16616-16621. doi:10.1073/pnas.1315235110

Feinberg, D.A., Moeller, S., Smith, S.M., Auerbach, E., Ramanna, S., Gunther, M., Glasser, M.F., Miller, K.L., Ugurbil, K., Yacoub, E., 2010. Multiplexed echo planar imaging for subsecond whole brain FMRI and fast diffusion imaging. PLoS One 5, e15710. doi:10.1371/journal.pone.0015710

Fischl, B., Sereno, M.I., Dale, A.M., 1999. Cortical surface-based analysis. II: Inflation, flattening, and a surface-based coordinate system. Neuroimage 9, 195-207. doi:10.1006/nimg.1998.0396

Friston, K.J., Williams, S., Howard, R., Frackowiak, R.S., Turner, R., 1996. Movement-related effects in fMRI time-series. Magn. Reson. Med. 35, 346-355. doi:10.1002/mrm. 1910350312

Goldstein, R.Z., Tomasi, D., Rajaram, S., Cottone, L.A., Zhang, L., Maloney, T., Telang, F., AliaKlein, N., Volkow, N.D., 2007. Role of the anterior cingulate and medial orbitofrontal cortex in preprocessing drug cues in cocaine addiction. Neuroscience 144, 1153-1159. doi:10.1016/j.neuroscience.2006.11.024 
Gorgolewski, K., Burns, C.D., Madison, C., Clark, D., Halchenko, Y.O., Waskom, M.L., Ghosh, S.S., 2011. Nipype: a flexible, lightweight and extensible neuroimaging data processing framework in python. Front. Neuroinform. 5, 13. doi:10.3389/fninf.2011.00013

Grill-Spector, K., Malach, R., 2004. The human visual cortex. Annu. Rev. Neurosci. 27, 649677. doi: 10.1146/annurev.neuro.27.070203.144220

Gröne, M., Dyck, M., Koush, Y., Bergert, S., Mathiak, K.A., Alawi, E.M., Elliott, M., Mathiak, K., 2015. Upregulation of the rostral anterior cingulate cortex can alter the perception of emotions: fMRI-based neurofeedback at 3 and 7 T. Brain Topogr. 28, 197-207. doi:10.1007/s10548-014-0384-4

Hagmann, P., Cammoun, L., Gigandet, X., Meuli, R., Honey, C.J., Van Wedeen, J., Sporns, O., 2008. Mapping the structural core of human cerebral cortex. PLoS Biol. 6, 1479-1493. doi:10.1371/journal.pbio.0060159

Jenkinson, M., Beckmann, C.F., Behrens, T.E.J., Woolrich, M.W., Smith, S.M., 2012. Fsl. Neuroimage 62, 782-790. doi:10.1016/j.neuroimage.2011.09.015

Kagan, J., 1966. Reflection-impulsivity: the generality and dynamics of conceptual tempo. J. Abnorm. Psychol. 71, 17-24. doi:http://dx.doi.org/10.1037/h0022886

Kelly, A.M.C., Di Martino, A., Uddin, L.Q., Shehzad, Z., Gee, D.G., Reiss, P.T., Margulies, D.S., Castellanos, F.X., Milham, M.P., 2009. Development of anterior cingulate functional connectivity from late childhood to early adulthood. Cereb. Cortex 19, 640-657. doi:10.1093/cercor/bhn117

Lavin, C., Melis, C., Mikulan, E., Gelormini, C., Huepe, D., Ibañez, A., 2013. The anterior cingulate cortex: an integrative hub for human socially-driven interactions. Front. Neurosci. 7, 64. doi:10.3389/fnins.2013.00064 
Lenartowicz, A., Mclntosh, A.R., 2005. The role of anterior cingulate cortex in working memory is shaped by functional connectivity. J. Cogn. Neurosci. 17, 1026-1042. doi:10.1162/0898929054475127

Li, N., Ma, N., Liu, Y., He, X.S., Sun, D.L., Fu, X.M., Zhang, X., Han, S., Zhang, D.R., 2013. Resting-state functional connectivity predicts impulsivity in economic decision-making. J. Neurosci 33, 4886-4895. doi:10.1523/JNEUROSCI.1342-12.2013

Margulies, D.S., Kelly, A.M.C., Uddin, L.Q., Biswal, B.B., Castellanos, F.X., Milham, M.P., 2007. Mapping the functional connectivity of anterior cingulate cortex. Neuroimage 37, 579-588. doi:10.1016/j.neuroimage.2007.05.019

Marques, J.P., Kober, T., Krueger, G., van der Zwaag, W., Van de Moortele, P.F., Gruetter, R., 2010. MP2RAGE, a self bias-field corrected sequence for improved segmentation and T1mapping at high field. Neuroimage 49, 1271-1281. doi:10.1016/j.neuroimage.2009.10.002

Mason, M.F., Norton, M.I., Van Horn, J.D., Wegner, D.M., Grafton, S.T., Macrae, C.N., 2007. Wandering minds: the default network and stimulus-independent thought. Science 315, 393-395. doi:10.1126/science.1131295

Moeller, F.G., Barratt, E.S., Dougherty, D.M., Schmitz, J.M., Swann, A.C., 2001. Psychiatric aspects of impulsivity. Am. J. Psychiatry 158, 1783-1793. doi:10.1176/appi.ajp.158.11.1783

Moeller, S., Yacoub, E., Olman, C.A., Auerbach, E., Strupp, J., Harel, N., Ugurbil, K., 2010. Multiband multislice GE-EPI at 7 tesla, with 16-fold acceleration using partial parallel imaging with application to high spatial and temporal whole-brain fMRI. Magn. Reson. Med. 63, 1144-1153. doi:10.1002/mrm.22361 
Nooner K.B., Colcombe S.J., Tobe R.H., Mennes M., Benedict M.M., Moreno A.L., et al., 2012. The NKI-rockland sample: a model for accelerating the pace of discovery science in psychiatry. Front. Neurosci. 6,152. doi:10.3389/fnins.2012.00152

Ochsner, K.N., Gross, J.J., 2005. The cognitive control of emotion. Trends Cogn. Sci. 9, 242249. doi:10.1016/j.tics.2005.03.010

Peters, J., Büchel, C., 2010. Episodic future thinking reduces reward delay discounting through an enhancement of prefrontal-mediotemporal interactions. Neuron 66, 138-148. doi:10.1016/j.neuron.2010.03.026.

Power, J.D., Barnes, K.A., Snyder, A.Z., Schlaggar, B.L., Petersen, S.E., 2012. Spurious but systematic correlations in functional connectivity MRI networks arise from subject motion. Neuroimage 59, 2142-2154. doi:10.1016/j.neuroimage.2011.10.018

Robbins, T.W., 2007. Shifting and stopping: fronto-striatal substrates, neurochemical modulation and clinical implications. Philos. Trans. R. Soc. Lond. B. Biol. Sci. 362, 917-932. doi:10.1098/rstb.2007.2097

Robbins, T.W., Gillian, C.M., Smith, D.G., de Wit, S., Ersche, K.D., 2012. Neurocognitive endophenotypes of impulsivity and compulsivity: towards dimensional psychiatry. Trends Cogn. Sci. 16, 81-91. doi:10.1016/j.tics.2011.11.009

Rokem, A., Trumpis, M., Perez, F., 2009. Nitime: time-series analysis for neuroimaging data, in: Varoquaux, G., van der Walt, S., Millman, J. (Eds), Proceedings of the 8th Python in Science Conference (SciPy 2009). Pasadena, pp. 68-75.

Schacter, D.L., Addis, D.R., 2007. The cognitive neuroscience of constructive memory: Remembering the past and imagine the future. Philos. Trans. R. Soc. Lond. B. Biol. Sci. 362, 773-786. doi:10.1098/rstb.2007.2087 
Shannon, B.J., Raichle, M.E., Snyder, A.Z., Fair, D.A., Mills, K.L., Zhang, D., Bache, K., Calhoun, V.D., Nigg, J.T., Nagel, B.J., Stevens, A.A., Kiehl, K.A., 2011. Premotor functional connectivity predicts impulsivity in juvenile offenders. Proc. Natl. Acad. Sci. U. S. A. 108, 11241-11245. doi:10.1073/pnas.1108241108

Sharma, L., Markon, K.E., Clark, L.A., 2014. Toward a theory of distinct types of "impulsive" behaviors: A meta-analysis of self-report and behavioral measures. Psychol. Bull. 140, 374-408. doi:10.1037/a0034418

Vogt, B.A., 2005. Pain and emotion interactions in subregions of the cingulate gyrus. Nat. Rev., Neurosci 6, 533-544. doi:10.1038/nrn1704

Weafer, J., Dzemidzic, M., Eiler, W., Oberlin, B.G., Wang, Y., Kareken, D.A., 2015. Associations between regional brain physiology and trait impulsivity, motor inhibition, and impaired control over drinking. Psychiatry Res. 233, 81-87. doi:10.1016/j.pscychresns.2015.04.010

Whiteside, S.P., Lynam, D.R., 2001. The five factor model and impulsivity: Using a structural model of personality to understand impulsivity. Pers. Individ. Dif. 30, 669-689. doi:10.1016/S0191-8869(00)00064-7

Winstanley, C.A., Eagle, D.M., Robbins, T.W., 2006. Behavioral models of impulsivity in relation to ADHD: Translation between clinical and preclinical studies. Clin. Psychol. Rev. 26, 379395. doi:10.1016/j.cpr.2006.01.001

Woldorff, M.G., Hazlett, C.J., Fichtenholtz, H.M., Weissman, D.H., Dale, A.M., Song, A.W., 2004. Functional parcellation of attentional control regions of the brain. J. Cogn. Neurosci. 16, 149-165. doi:10.1162/089892904322755638

Woodward, T.S., Metzak, P.D., Meier, B., Holroyd, C.B., 2008. Anterior cingulate cortex signals the requirement to break inertia when switching tasks: A study of the bivalency effect. Neuroimage 40, 1311-1318. doi:10.1016/j.neuroimage.2007.12.049 
Yantis, S., Anderson, B.A., Wampler, E.K., Laurent, P.A., 2012. Reward and attentional control in visual search. Nebr. Symp. Motiv. 59, 91-116.

Yarkoni, T., Poldrack, R.A., Nichols, T.E., Van Essen, D.C., Wager, T.D., 2011. Large-scale automated synthesis of human functional neuroimaging data. Nat. Methods $8,665-670$. doi:10.1038/nmeth.1635. 


\section{seed}

MPI-S

NKI-RS

s1

ही जि

किते है।

कित)

का?

बiा

Eases

s7

End

है।

5.

है।

हn

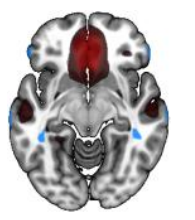

i9

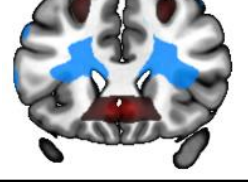

and

बित्र हो :

कात 213

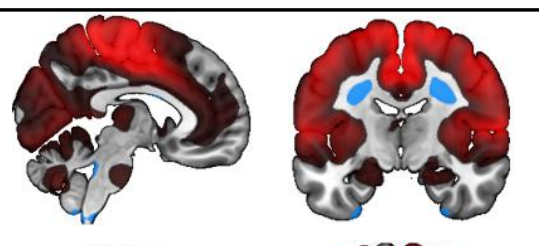

हा?

हुare है।
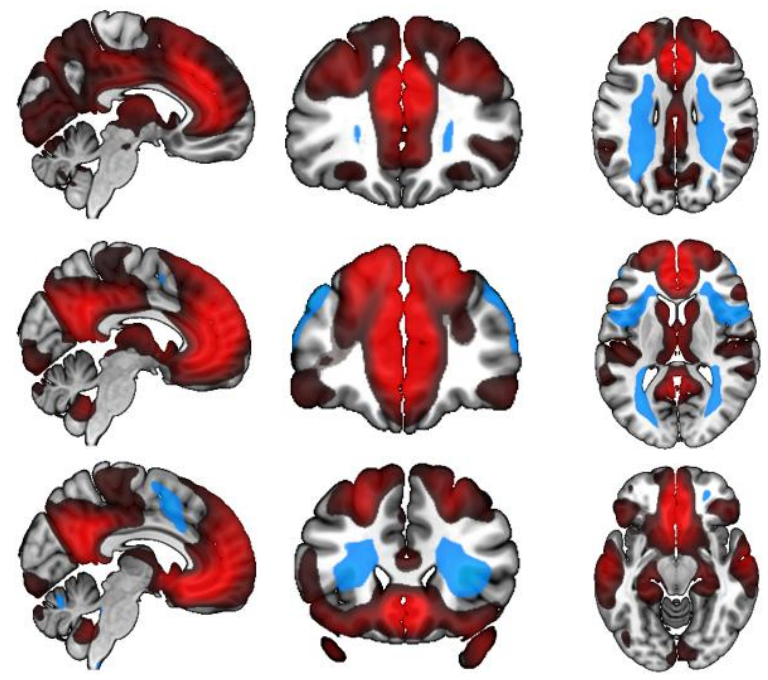

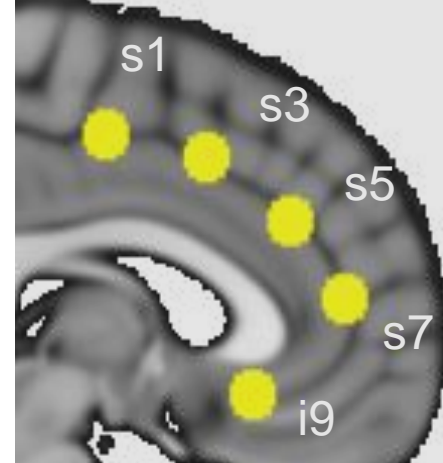

s1 +/- 5, -10, 47

s3 $+/-5,14,42$

s5 +/- 5, 34, 28

s7 $+/-5,47,11$

i9 $+/-5,25,-10$

.1 


\section{positive urgency}

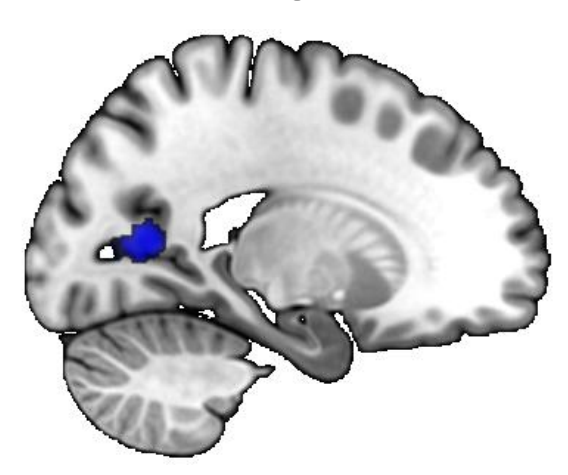

$-20$
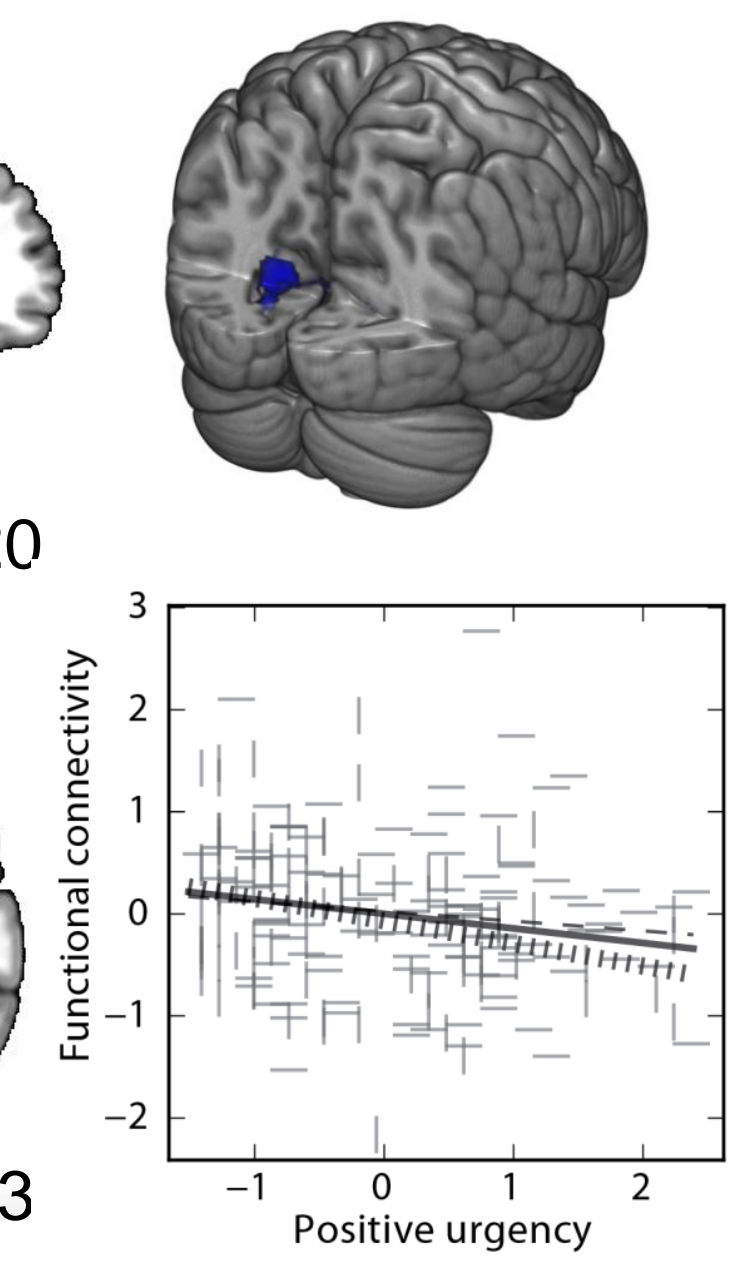

Seed
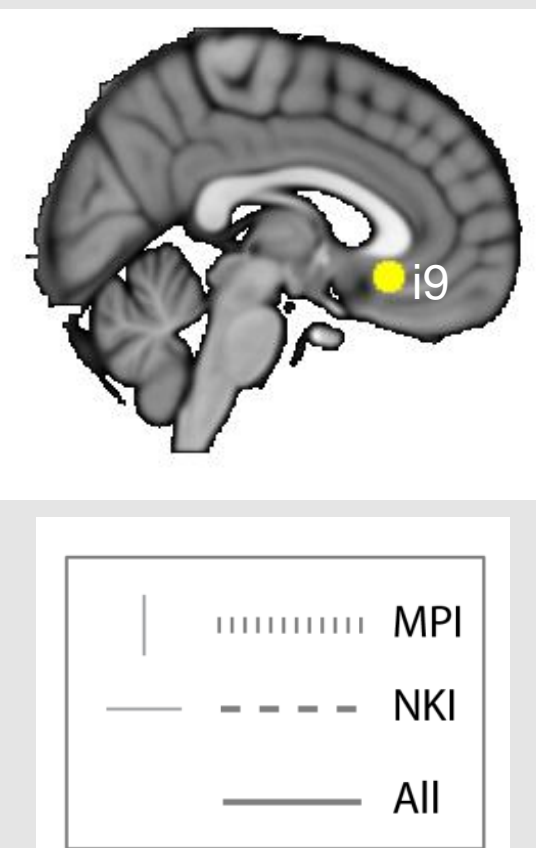

Z

0.1

$$
z=3.1, p=0.005
$$


Lack of perseverance

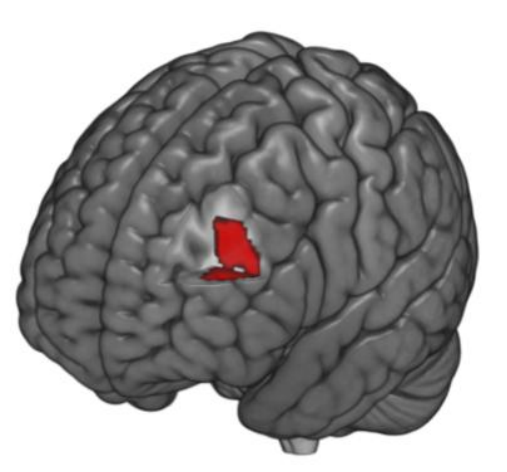

Lack of premeditation

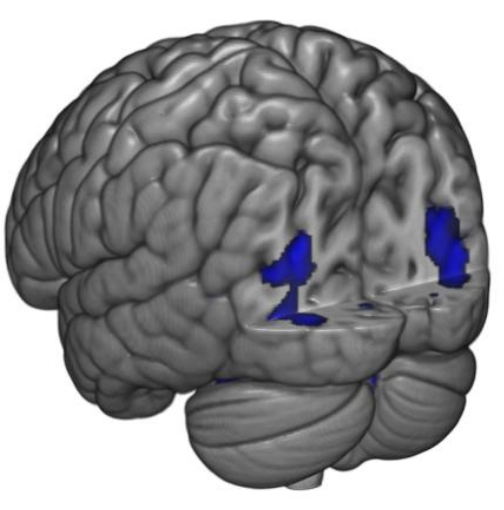

Seed
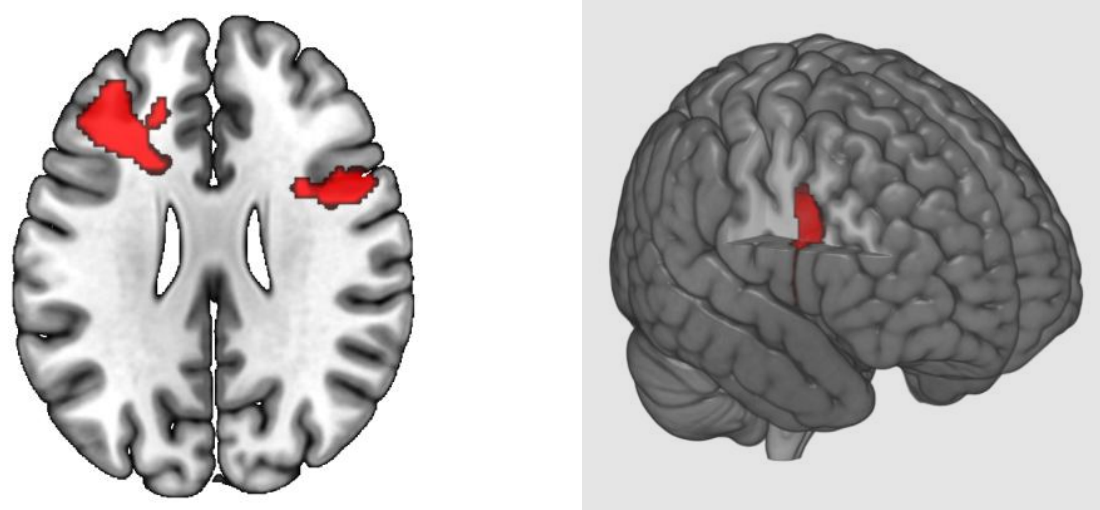

$+27$

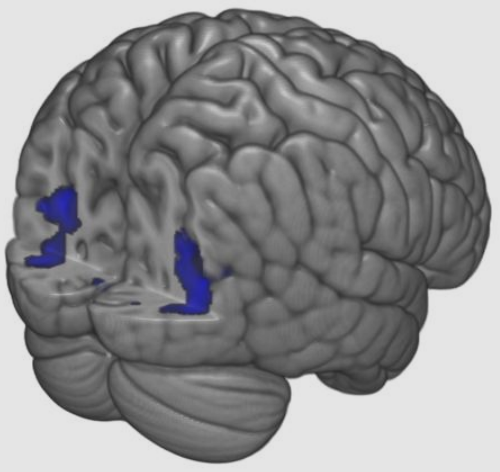

Z

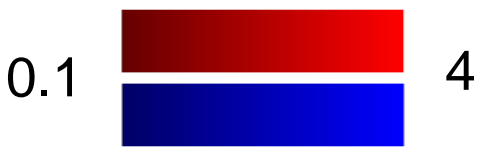

$z=3.1, p=0.005$

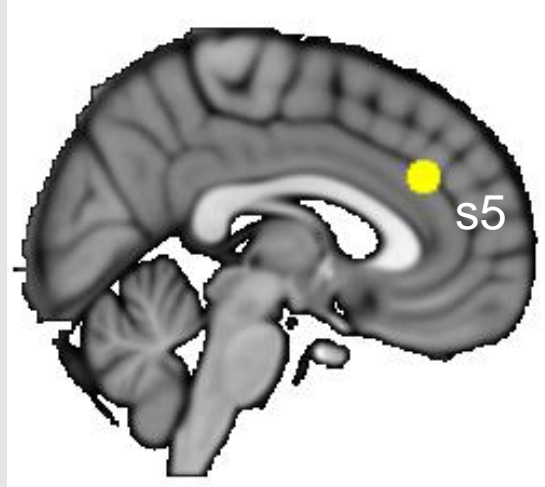



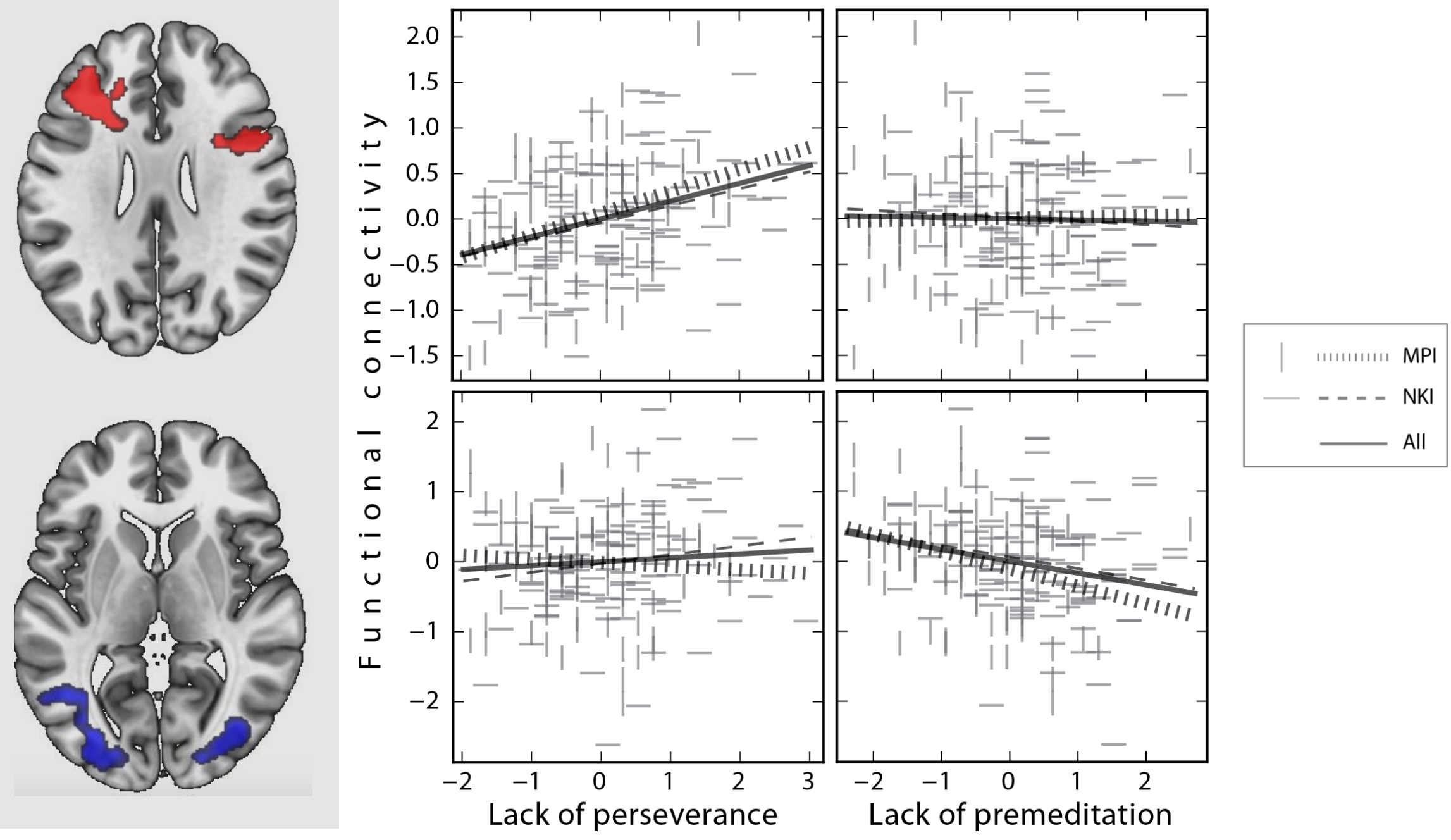

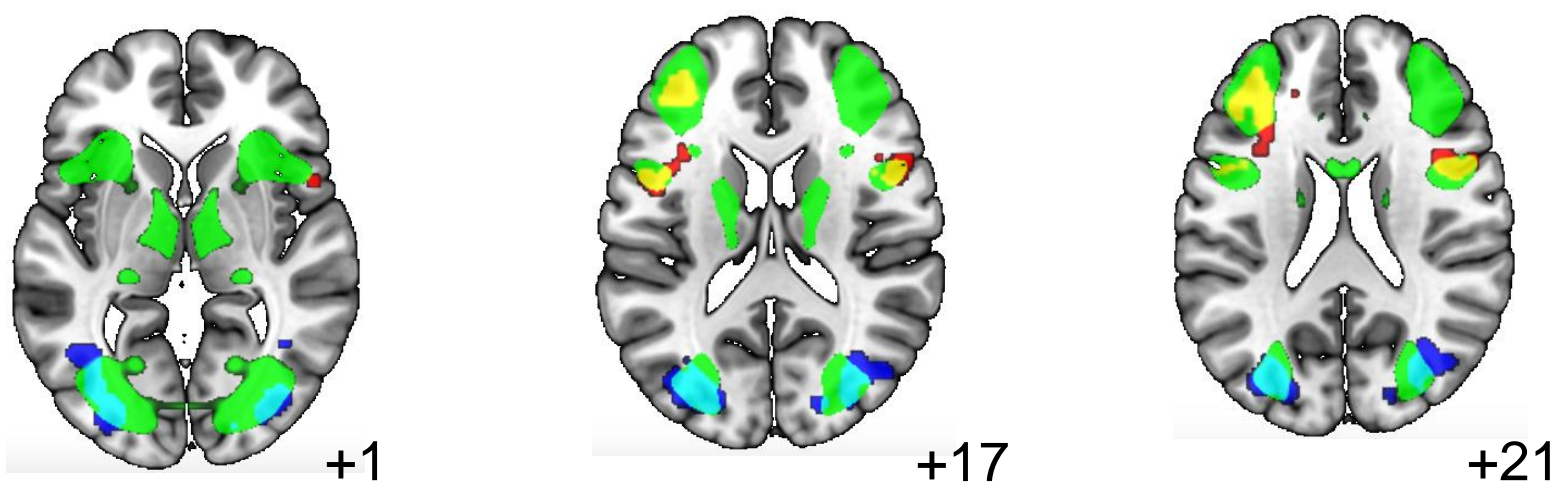

Poor

Poor

premeditation perseverance
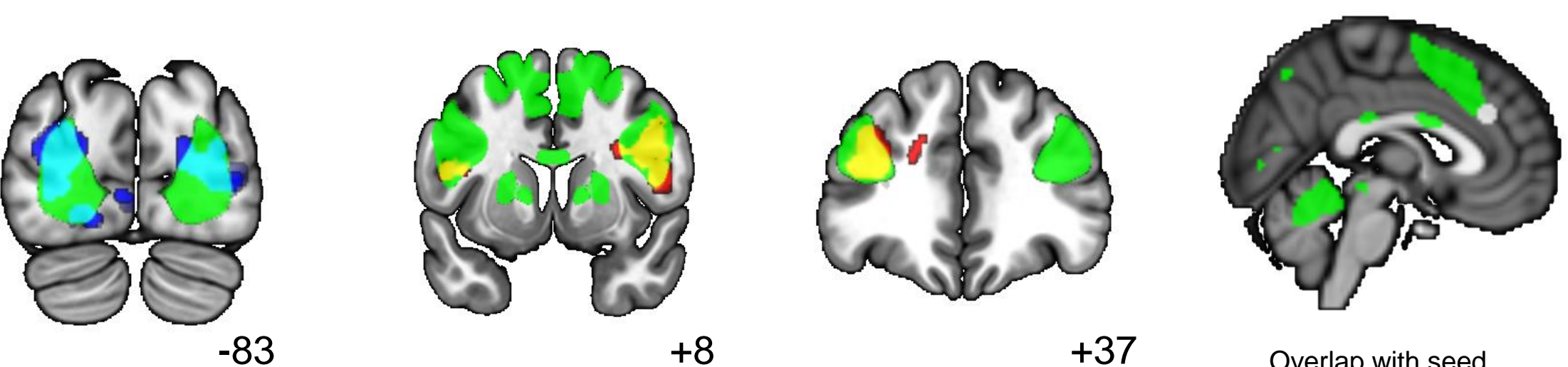

$+8$

$+37$

Overlap with seed

Multiple Demand Network 